\title{
The Distance to the Pleiades Revisited
}

\author{
David Valls-Gabaud \\ GEPI, CNRS UMR 8111, Observatoire de Paris, France \\ email: david.valls-gabaud@obspm.fr
}

\begin{abstract}
The parallax of the Pleiades has been mired in controversy ever since the very first astrometric measures in the late 1880s. Over a century later, the measures from the HIPPARCOS catalogue gave results which were inconsistent with the distance inferred from the fitting of the colour-magnitude diagram. We briefly review here the debate and focus on the various attempts made at solving the problem, and especially those using binary stars. The only double-lined eclipsing binary found so far in the Pleiades, HD 23642, provides not only the final answer to the problem but also, through detailed state-of-the-art analyses, the fundamental calibration for binaries in more distant clusters and hence in the Local Group. We discuss some of the various sources of systematic uncertainties that limit, so far, the accuracy of the measured stellar parameters to about $1 \%$, and the progress that is required to break this barrier.
\end{abstract}

Keywords. Stars: fundamental parameters, Stars: binaries: eclipsing, Stars: binaries: spectroscopic, Galaxy: open clusters, Methods: statistical

\section{Introduction}

[...] the project of determining the parallax of the Pleiades is not altogether hopeless.

Agnes M. Clerke (1893)

The distance to the Pleiades has a long, distinguished and painful history. Even though the first attempts at measuring the parallax of its members with photographic plates resulted in widely different results, the optimism of Clerke (1893) seemed reasonable at the time. However, over a century later, and in spite of the tremendous progress made, some pessimism seems to remain:

I have been in astronomy so long that I don't really believe astronomical distances are much good.

Donald Lynden-Bell (1998)

In the context of the cosmological distance scale, pinning down the distances of nearby clusters is essential to calibrate the primary, and then the secondary, distance indicators. The largest contribution to the systematic uncertainty in the measurement of the Hubble parameter $H_{0}$ comes from the uncertainties in the distance to the Large Magellanic Cloud, which in turn reflect, in part, the systematics in the primary calibrations. Measuring locally $H_{0}$ to within a systematic uncertainty below $3 \%$ would allow us to constrain, for instance, the equation of state of the dark energy field that seems to accelerate the expansion of the universe.

Over time, and quite remarkably, the trigonometric parallaxes of the Pleiades have systematically decreased, yielding historically increasing distances from 27 pc (Schouten 1919), 83 pc (Alden 1923), 101 pc (Binnendijk 1946) and 130 pc (van Leeuwen 1983), a distance which appeared to agree with the photometric parallaxes inferred from its colour-magnitude diagram. 


\section{HIPPARCOS vs Stellar Physics?}

The parallax of the Hyades inferred from HIPPARCOS (Perryman et al. 1998) showed that the half-mass radius was a significant fraction $(\sim 12 \%)$ of the distance, and hence depth effects are important when interpreting the colour-magnitude diagram, and especially when testing stellar evolution models. More distant similar clusters should provide much better tests, and it came as a surprise that the HIPPARCOS parallax was significantly larger than the one inferred from photometric parallaxes (van Leeuwen \& Hansen-Ruiz 1998, Mermilliod et al. 1997, Robichon et al. 1999). The difference is very significant, and amounts to some $13 \mathrm{pc}$, that is, around 1 milli-arcsecond or -0.3 magnitudes (Pinsonneault et al. 1998). The error in the HIPPARCOS zero point amounting to less than 0.1 mas (Arenou et al. 1995, Lindegren 1995), the controversy started in earnest. Either stellar evolution had to be modified to account for this new distance, or there are unaccounted problems in the HIPPARCOS catalogue, or both.

Changes suggested for the stellar tracks included an increase in the helium abundance, up to $Y=0.34$ (Belikov et al. 1998) and a decrease in metallicity, down to $Z=0.012$ (Castellani et al. (2002). Although a photometric abundance of $[\mathrm{Fe} / \mathrm{H}]=-0.1$ was claimed (Grenon 2002), precise spectroscopic abundances range from 0.026 (Boesgaard \& Friel 1990) to 0.13 (Cayrel et al. 1988) through 0.04 (King et al. 2000). It would therefore be highly surprising that the helium abundance would be as large as 0.34 , given that the Hyades, at $[\mathrm{Fe} / \mathrm{H}]=0.14$, has an inferred $Y=0.26 \pm 0.02$. On the other hand, there are hardly any spectroscopic measures of the helium and iron abundance in clusters, and hence the dispersion may be very large. As van Leeuwen (1999) observed, in clusters with well-measured HIPPARCOS parallaxes, there seems to be a trend in age in their colour-magnitude diagram that appears to be unexplained by current stellar evolutionary tracks. The most recent, and accurate, colour-magnitude diagram (CMD) for the Pleiades has been made by An et al. (2006). In the $B-V$ diagram, the lower main-sequence stars appear far too blue in comparison with the predicted isochrone. This may perhaps be explained by their chromospheric activity (even though in the $V-I_{c}$ and $V-K_{s}$ diagrams they appear normal). In contrast, it is in the $V-I_{c}$ diagram of M67, where stars appear to be bluer than predicted. To make different CMDs consistent with the same distance, an empirical correction has to be applied to each CMD and to each cluster. It is therefore only the upper, brighter half of the CMD that seems safe from strong systematic trends, a conclusion also reached by Percival et al. (2005), who showed that the optical CMDs required an implausible low $[\mathrm{Fe} / \mathrm{H}]=-0.4$, while the IR CMDs are consistent above $M_{V}=6$ with a distance of $134( \pm 3)$ pc. Changes in the shapes of the main sequence may also arise from variations in the mixing length parameter, which usually is assumed to be constant at all masses, ages and metallicities, while there may be tentative evidence of the contrary (Lastennet et al. 2003).

There are also more indirect ways of constraining the distance. For instance, Fox Machado et al. (2006) use measured seismological modes along with assumed ages and metallicities to produce consistent values for the distance. Unless better constraints for each star are used, this purely astrophysical method does not give yet strong constraints to be compared to the photometric parallax.

Ever since the very first HR diagram, by Hans Rosenberg (1911) $\dagger$ the Pleiades have been used as a calibrator and many of the distances inferred have found the value of the calibration. There is therefore much space for a revised distance, and the HIPPARCOS parallax has no reason to be, a priori, rejected. This is especially true given that the other method to measure distances, using the convergent point, is not ideal in the Pleiades,

$\dagger$ and not by Hertzsprung or Russell. 
since the radial velocity is small and hence the expansion-related proper motion at large distances from the centre is small and there are few stars to measure. At any rate, the effect is roughly similar to the intrinsic velocity dispersion and makes any distance estimate highly uncertain.

Ground-based trigonometric parallaxes, on the other hand, yield values which appear systematically smaller than the ones from HIPPARCOS, even though the differences are within $1 \sigma$ (Gatewood et al. 2000).

Could the HIPPARCOS parallaxes be in error, as claimed in various papers (e.g., Makarov 2002)? Part of the problem resides in the fact that the HIPPARCOS-inferred distance does not come from the straight average of the distances of stars which are members of the clusters, but rather comes as a global solution to parallaxes and proper motions based on the individual abscissa residuals. Correlations between abscissae measured within each data set, reduced independently by the two consortia (NDAC and FAST), are now taken into account (van Leeuwen 2004) but the inferred proper motions are inconsistent with accurate ground-based measures. This indicates that even the revised parallax from Hipparcos, at about 8.0 mas, is subject to caution. It may well be that the global solution for the cluster is actually not properly attached to the overall solution, and that there may be local zero-point offsets. The stars of the Pleiades being so bright, the error in the abscissa residuals come from the attitude of the satellite, rather than photon noise (which dominates at the fainter magnitudes). A full, new reduction taking into account all these effects is currently been completed (van Leeuwen 2004).

Could a different trigonometric-based parallax be made? For instance, using the Fine Guidance Sensor onboard HST, Soderblom et al. (2005) found that the 3 stars they observed had parallaxes inconsistent (within 1.5 $\sigma$ ) with HIPPARCOS. Note however that the HST-based parallaxes are differential, that is, measured with respect to background stars which have their own parallaxes. One has to assume their distances based on spectral types and absolute magnitudes, and iteratively find a consistent solution for both background and foreground stars. In other words, one relies on calibrations using stellar physics, and so the argument is somewhat circular, as it is not very surprising to find that the 3 Pleiades stars measured by Soderblom et al. (2005) do agree with the distance inferred from the fitting of the CMD with stellar isochrones.

\section{Binaries to the Rescue}

Remarkably, binaries can solve the controversy, as they may bring a fully stellar physics independent way of measuring distances. The first of class of binaries which comes to mind that can yield distance measurements is the interferometric one. Atlas, one of the "seven" (or is it six?) bright stars that make the naked-eye Pleiades, was known to be an interferometric binary and observations carried out at the Mark III interferometer in 1989-1992 and then at the PTI (1996-1999) by Pan et al. (2004) gave a distance between 133 and 137 pc. However, they had to assume a mass-luminosity relation to get these values, and we are again in a case of circular reasoning: astrophysical inputs are likely to yield the photometric parallax... Using 12 further astrometric measures at the Mark III and NPOI, and, crucially, spectra, Zwahlen et al. (2004) succeeded in getting a purely geometrical distance to Atlas of $132 \pm 4 \mathrm{pc}$. A note of caution comes from the fact that the spectra had to be disentangled in order to produce a radial velocity curve for both components, and the low amplitude of the curve $(37$ and $26 \mathrm{~km} / \mathrm{s})$ means that there is room for improvement. Nevertheless, this was a superb achievement and further measures on other such binaries would be welcome, especially to assess the depth of the cluster. The individual HIPPARCOS parallax for Atlas is not that discrepant with this value. 


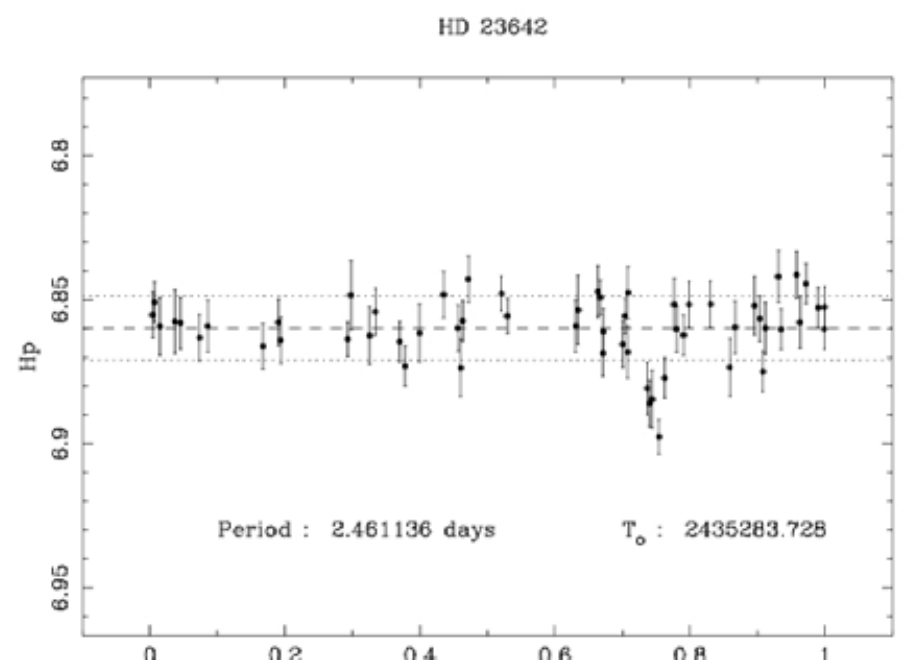

Figure 1. The HIPPARCOS light curve, folded with the period found by Griffin (1995), showing the secondary eclipse. HIPPARCOS managed to miss the primary eclipse completely, while the secondary eclipse is sampled with 5 points.

The second class of binaries that are important in this context is the one formed by double-lined (SB2), eclipsing binaries. In this case, not only the full orbital solution can be computed, but also the physical one, yielding the distance with no astrophysical calibrations whatsoever. Peering through the catalogues of SB2 binaries, there are only four of them in the Pleiades. Using the periods measured from the radial velocity curves, we folded, back in November 1997, the HIPPARCOS light curves and found, much to our delight, that HD 23642 had eclipses, as predicted by Griffin (1995). Unfortunately the sampling of HIPPARCOS was less than ideal in that it missed completely the primary eclipse (Figure 1) ! This was independently found by Torres (2003) as well.

Even though the eclipse is very shallow, denoting a grazing configuration, the star is very bright and serious amateurs could produce detailed light curves. An international observing campaign was launched, resulting in the detection of the primary eclipse with a depth of $0.08 \mathrm{mag}$, and the confirmation of the 0.05 mag-deep secondary eclipse (Miles 1999) both with CCD and photoelectric detectors. The star is so bright $\left(H_{p}=6.84\right)$ that professional instruments are unable to monitor it, and the role of amateurs has been essential in securing fully-sampled multi-band light curves.

Incidentally, the first "professional" observations of HD 23642 were made by Galileo (1610) in January-February 1610 (Figure 2). Given the importance of this binary for the distance scale determination, we have been securing high-precision light curves and spectra to make this binary the primary calibrator for eclipsing SB2s.

\section{HD 23642 and the distance of the Pleiades}

HD 23642 was already singled out by Giannuzzi (1985) who attempted to infer its distance through astrophysical calibrations, assuming an age and composition. This approach has a number of problems (e.g., Lastennet \& Valls-Gabaud 2002). Munari et al. (2005) took five spectra and combined these new radial velocities with the old values measured in photographic plates, along with some 500 and 430 photometric measurements in the $B$ and $V$ bands respectively. They measured the effective temperature of the primary to be $9671 \pm 46 \mathrm{~K}$, and the secondary at $8023 \pm 544 \mathrm{~K}$, which yielded the 


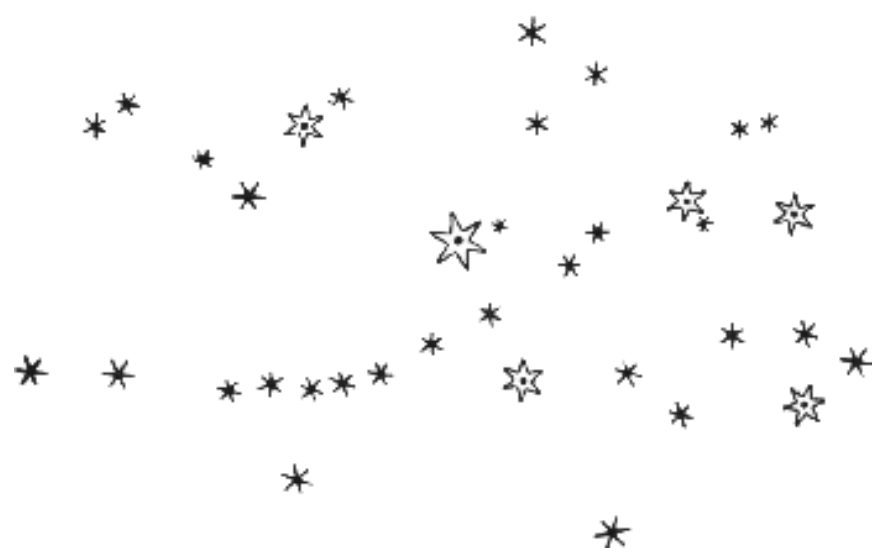

Figure 2. The first observations of HD 23642 were carried out by Galileo in 1610 as part of his quick survey of the winter sky.

absolute magnitudes and hence a distance of $132 \pm 2 \mathrm{pc}$. This determination, with a precision of $1.5 \%$, depends critically on the adopted reddening of $\mathrm{E}(\mathrm{B}-\mathrm{V})=0.012$. This is an implausibly small value, even knowing the strongly differential reddening of the cluster. Southworth et al. (2005) analysed the same data set as Munari et al. (2005), and, even adopting the same reddening, found more realistic values for the effective temperatures $(9750 \pm 250 \mathrm{~K}$ and $7600 \pm 400 \mathrm{~K})$ yielding a larger distance of $139 \pm 3 \mathrm{pc}$ and widening the difference with respect to the HIPPARCOS distance of $111 \pm 12 \mathrm{pc}$ for this binary. A crucial aspect of their work was to realise that systematics may dominate the error budget and they explored this problem using extensive Monte Carlo simulations of synthetic light curves at their best solution. Unfortunately they kept fixed the mass ratio to the value found spectroscopically, so the cross-talk between the radial velocity curves and the light curves was not explored. In addition, the peculiar nature of the secundary, an Am star, makes both the interpretation of the light curves and effective temperature more uncertain (Burkhart \& Coupry 2000, Hui-Bon-Hoa 2000, Böhm-Vitense 2006).

Our approach has been to keep under control as much as feasible the systematic errors. Following the well-established HIPPARCOS tradition, we will not give preliminary results, but will describe part of the work that we have been doing over the past eight years on this unique binary. First the photometry. Systematics being different at different telescopes, we secured light curves in the same photometric filters at different locations and telescopes. Next, we covered the optical light curves observing in the $U B V R I$ filters, with a fourfold redundancy in $V$ and twice in $I$. In total, we made 12382 photometric observations (Figure 3). This dataset allows us to constrain both model atmospheres and limb darkening (e.g., Claret \& Hauschildt 2003).

Second, we also took spectra at different telescopes/instruments/resolutions and made a careful analysis of the zero point offsets, along with the influence of weigths given to each observation. The resulting radial velocity curve has over 80 new spectra with well understood systematics (Figure 4).

Third, we secured very high resolution and signal-to-noise spectra at both the CFHT (using Gecko and Espadons) and VLT (UVES) so that a detailed abundance analysis of each component can be carried out (Figure 5). Similarly, the spectra taken with different telescopes allow us to understand the observed variations in the line profiles through Fourier analysis. The realisation that Vega is a rapid, pole-on rotator (Peterson et al. 


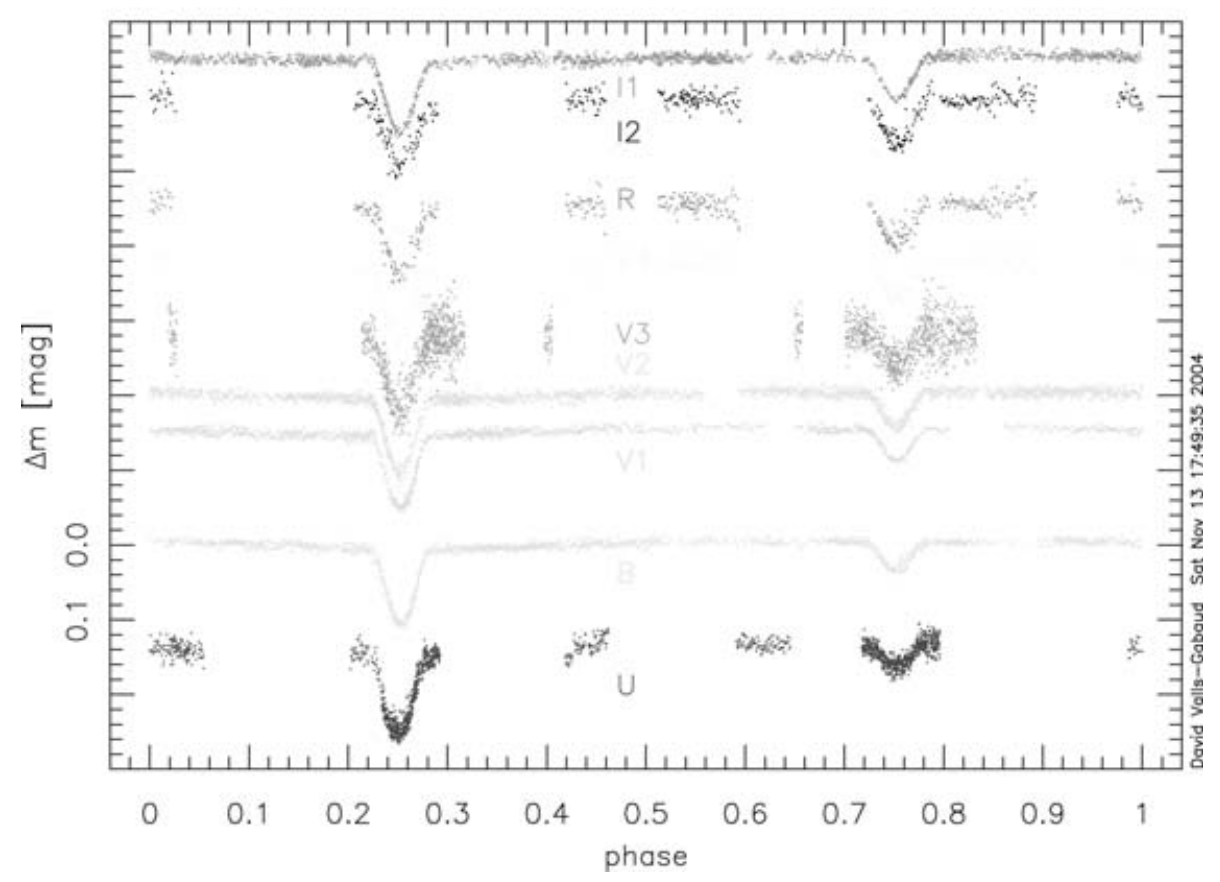

Figure 3. Summary of our 12382 photometric observations over the $U B V R I$ filters carried out over the past 7 years at different locations/telescopes to assess systematics properly.

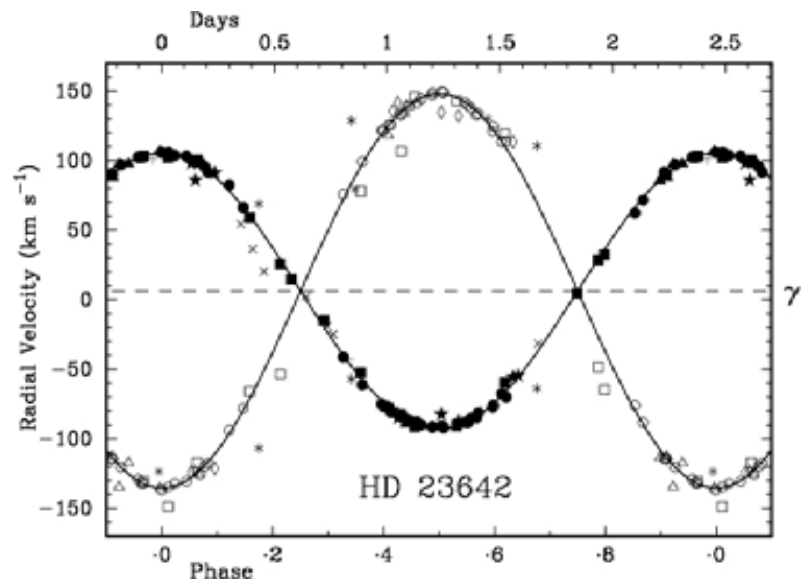

Figure 4. Combined radial velocity curves with modern and old spectra.

2006) provides yet another cautionary tale. The interstellar absorption lines over this wide wavelength range, combined with spectra of other stars in the Pleiades, allow us to map the gas that may be tracing the dust content along the line of sight (e.g., White et al. 2001).

Finally, we combined the optical spectra with 2MASS IR photometry and IUE SWP and LWP spectra to set constraints on the full spectral energy distribution spanning the $1000 \AA-2.2 \mu \mathrm{m}$ range (Figure 6 ).

This unique dataset allows us to address a number of problems in quantifying the systematic errors: (1) zero points in radial velocities, (2) differential reddening due to the 

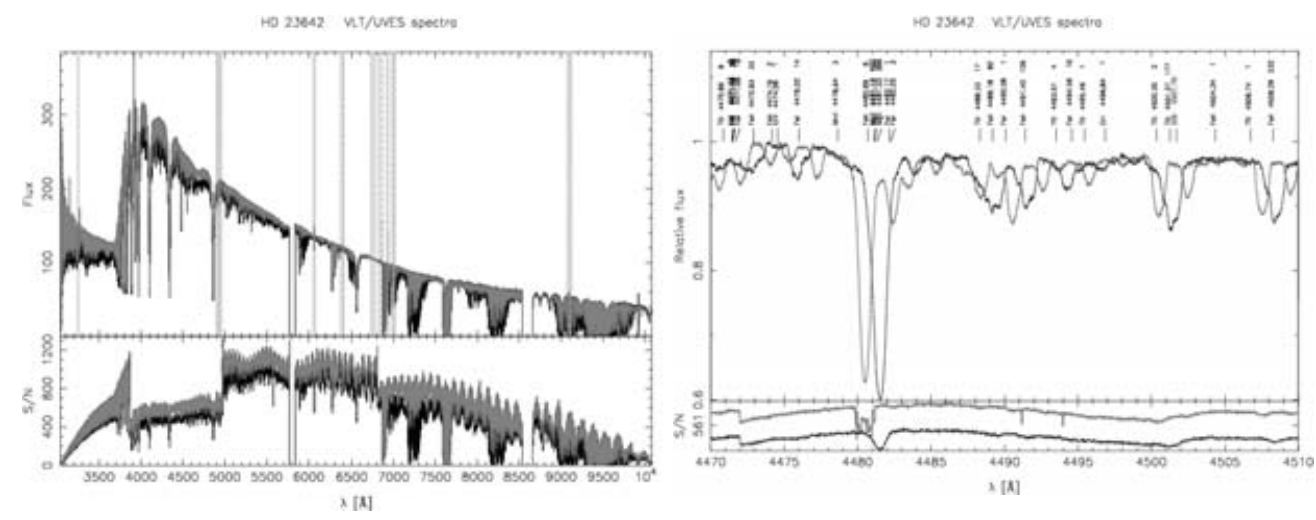

Figure 5. (Left) UVES spectra taken at the VLT spanning the range $3400-9,500 \AA$ with an overall signal-to-noise ratio larger than 400 . The shaded regions mark areas with problems in the detector. Note also the gaps at 5800 and $8600 \AA$. (Right) Zoom in the $4490 \AA$ with an average $\mathrm{S} / \mathrm{N}=560$. The identifications and EWs in the rest frame are marked.

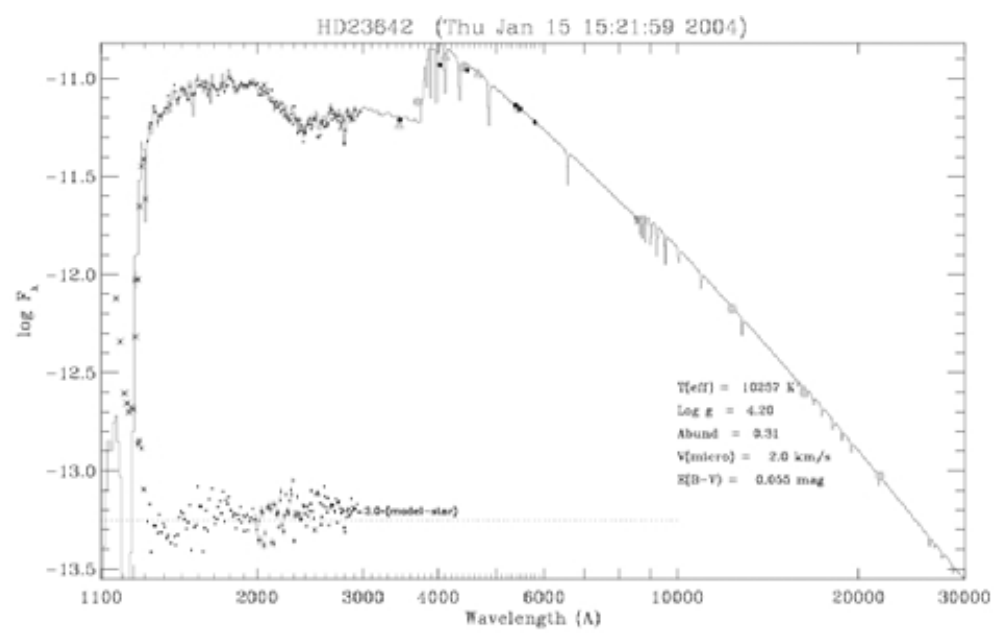

Figure 6. Overall spectral energy distribution, using IUE, optical and 2MASS data.

patchy extinction, (3) the effect of diffuse light due to the reflection nebula, and possible third light contribution, (4) the effects of the assumed limb darkening, and (5) the effects of assumptions regarding model atmospheres.

\section{Summary}

Although the distance to HD 23642 is not quite settled yet, we are on the right path to quantify properly both the statistical and systematic errors with unprecedented detail. This binary will prove to be the fundamental calibration for eclipsing, double-lined spectroscopic binaries, and will be essential for studies of these binaries in the Local Group. The parallax of the Pleiades is not an hopeless project, as Agnes Clerke correctly pointed out, but truly breaking the $1 \%$ barrier in precision and accuracy does require more hard work than anticipated.

\section{Acknowledgements}

I would like to thank my collaborators in this 8 year-long project: I. Ribas, G. Torres, R.F. Griffin, E. Fitzpatrick, F. Royer, J.M. Gómez Forrellad, E. García Melendo, C.H. 
Lacy and D. Terrell. Thanks are also due to the members of the Variable Star Section of the British Astronomical Association, and especially R. Miles, J. Saxton, and K. Holland.

\section{References}

Alden, H.L. 1923, AJ 35, 61

An, D., et al. 2006, ApJ in press

Arenou, F., et al. 1995, A\&A 304, 52

Belikov, A.N. et al. 1998, A\&A 332, 575

Binnendijk, L. 1946, Ann. Sterrew. Leiden 19, 2

Boesgaard, A.M., Friel, E.D. 1990, ApJ 351, 467

Böhm-Vitense, E. 2006, PASP 118, 419

Burkhart, C., Coupry, M.F. 2000, A\&A 354, 216

Castellani, V. et al. 2002, MNRAS 334, 193

Cayrel, R. et al. 1988, in IAU Symp 132, 449

Claret, A., Hauschildt, P.H. 2003, A $\mathscr{E} A$ 412, 241

Clerke, A.M. 1893, Observatory 16, 198

Fox Machado, L. et al. 2006, A\&SA 446, 611

Galileo, G. 1610, Sidereus Nuncius (Florence)

Gatewood, G. et al. 2000, ApJ 533, 938

Giannuzzi, M.A. 1995, A\&A 293, 360

Grenon, M. 2002, Highlights in Astronomy 12, 680

Griffin, R.F. 1995, JRASC 89, 53

Hui-Bon-Hoa, A. 2000, A\&AS 144, 203

King, J.R. et al. 2000, ApJ 533, 944

Lastennet, E. et al. 2003, A\&A 409, 611

Lastennet, E. \& Valls-Gabaud 2002, A\&A 396, 551

Lindegren, L. 1995, A\&A 304, 61

Lynden-Bell, D. 1998, in IAU Symp. 192, 15

Makarov, V.V. 2002, AJ 124, 3299

Mermilliod, J.C. et al. 1997, in Hipparcos Venice '97, ESA SP-402, 643

Miles, R. 1999, J. Brit. Astron. Assoc. 109, 106

Munari, U., et al. 2004, A\&SA 418, L31

Pan, X., Shao, M., Kulkarni, S.R. 2004, Nature 427, 326

Percival, S.M. et al. 2005, A\&\&A 429, 887

Perryman, M.A.C. et al. 1998, A\&SA 331, 81

Peterson, D.M. et al. 2006, Nature 440, 896

Pinsonneault, M.H., et al. 1998, ApJ 504, 170

Robichon, N., et al. 1999, A\&\&A 345, 471

Rosenberg, H. 1911, Ast. Nach. 186, 71

Schouten, W.J.A. 1919, Observatory 42, 240

Soderblom, D.R. et al. 2005, AJ 129, 1616

Southworth, J. et al. 2005, A\&\&A 429, 645

Tomkin, J. 2005, in ASP Conf. Series, 336, 199

Torres, G. 2003, IBVS 5402, 1

Van Leeuwen, F. 1983, Ph.D. Thesis, Leiden Univ.

Van Leeuwen, F. 1999, A\& A 341, L71

Van Leeuwen, F. 2004, in Transits of Venus, IAU Coll. 196, 347

Van Leeuwen, F., Hansen-Ruiz, C.S. 1997, in Hipparcos Venice '97, ESA SP-402, 689

White, R.E. et al. 2001, ApJ 132, 253

Zwahlen, N. et al. 2004, A\& A 425, L45 


\section{Discussion}

ViRginia TRImble: So I guess the question is: What went wrong with the Hipparcos parallaxes ?

VALLS-GABAUD: There is nothing fundamentally wrong with the parallaxes provided by HIPPARCOS! As a matter of fact, the comparison of accurate, parallax- and stellar physics- independent distances and the Hipparcos-inferred ones yield a perfect correlation within 50 pc (Tomkin 2005) and at larger distances the dispersion increases, exactly as one expects. The trouble may arise when computing average distances of extended clusters, from a handful of bright stars (Van Leeuwen 2004). 